\title{
Collision-Induced Dissociation
}

National Cancer Institute

\section{Source}

National Cancer Institute. Collision-Induced Dissociation. NCI Thesaurus. Code C48061.

A method by which frag mentation can be achieved. CID is accomplished by selecting an ion of interest with the mass analyzer and then subjecting that ion to collisions with neutral atoms or molecules. The resulting fragment ions are then mass analyzed. (from The Expanding Role of Mass Spectrometry in Biotechnology by Gary Siuzdak) 